\title{
A Novel Technique of Draping for Otologic Surgery: Mitigation of Aerosol Generation During Covid Pandemic
}

\author{
Naresh K. Panda ${ }^{1}\left(\mathbb{D} \cdot\right.$ Ganesh Agarwal $^{1} \cdot$ Neemu Hage $^{1} \cdot$ R. Balaji $^{1}$
}

Received: 18 June 2020/Accepted: 16 July 2020/Published online: 22 July 2020

(C) Association of Otolaryngologists of India 2020

\begin{abstract}
The surgical procedures in the temporal bone have the potential to generate aerosols which could affect the health care personnel. An innovative way to avoid aerosolization during ear surgery has been highlighted in this manuscript.
\end{abstract}

Keywords Aerosol generating surgery . Covid 19 pandemic $\cdot$ Draping technique

\section{Introduction}

The Covid-19 pandemic has posed challenges for the healthcare system all over the world. The otolaryngologists are constantly at risk of being exposed to aerosols during surgical procedures. Otological procedures particularly those requiring drilling of the temporal bone, have the potential of generating significant amount of aerosols. Aerosols, can carry viral particles up to several hours, thereby, having the potential to infect any individual within the confines of that space.

We have come up with a novel technique of draping for the purpose of performing otological surgeries, which is simple to execute and makes use of routine components used conventionally in surgeries. It was employed for subtotal temporal bone resection for a case of squamous cell carcinoma of the temporal bone. The patient was tested negative for Covid 19 before being taken up for surgery as per the Institute Protocol.

Naresh K. Panda

npanda59@yahoo.co.in

1 Departmant of Otolaryngology, PGIMER, Chandigarh, India

\section{Method}

The patient was laid supine on the OT table. Intubation was done under full precautions by an experienced anaesthetist. The head of the patient was then set to the desired position. Draping of the of the patient was done with surgical drapes. The microscope was draped as per the standard protocol. An instrument tray, which is an accessory attachment of the operative table, was attached to the table, on one side of the surgeon, depicted as 'A' in Fig. 1a. A Gottingen laser support table for laryngoscope holder $8575 \mathrm{~K} / \mathrm{KC}$, was then attached at the head end of the table, depicted as ' $\mathrm{B}$ ' in figure-1a. Both the instrument tray and the laser support table were draped with sterile cloth drapes secured with towel clips. The vertical height of both the stands was adjusted sufficiently above the level of the head of the patient. A steri-drape $(3 \mathrm{M})$ with its adhesive surface facing downwards was positioned between the two stands creating a bridge between them, as shown in Fig. 1b.

The microscope was then brought forward, positioned between the two draped stands and the operative field was focussed. Following this, a cut was made in the centre of the steri-drape to accommodate the microscope lens cap. Two sterile transparent surgical drapes were then used to create a tent-like structure by sticking the centre of each polydrape to the two free adhesive ends of the steri-drape. The loose flaps of the polydrapes were secured to the stands with sterile adhesive stickers to create a closed impermeable framework. By insinuating the hands below the surgical drapes, two corridors intended for the surgeon's and the nursing assistant's manoeuvrability was created. The final assembly was checked again to ensure that none of the bone dust and aerosol was allowed to move out of the confines of this closed compartment Fig. 2. The surgery was then executed. After the procedure, doffing of 


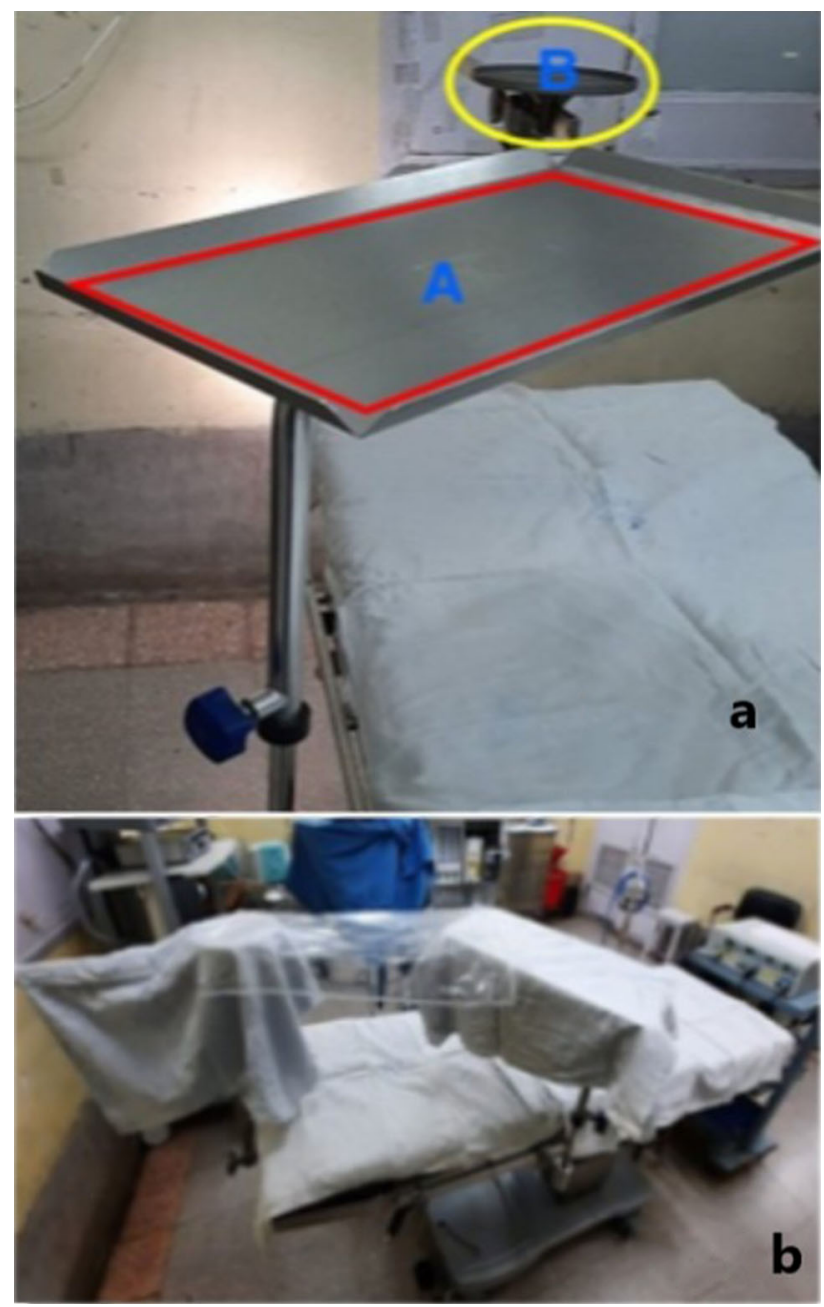

Fig. 1 a Showing the attachment of instrument tray (A) and Gottingen Laser support (B) to the operating table. b Showing the steridrape attached between the two attachments A and B

the draping assembly was done. It was ensured that the inner side of the drapes did not come in contact with any surface. Waste management was then carried out as per biomedical waste management guidelines.

\section{Discussion}

This innovative idea to mitigate aerosolisation of the operative field prevents exposure to the health personnel. This assembly of draping allowed adequate visualisation and surgeon manipulation as transparent drapes were utilised. No loosening of the drapes were observed and owing to the secure configuration provided by the steri-drape and the sterile adhesive stickers. The steri-drape was stretchable to a certain degree which allowed minor adjustments of the microscope throughout the surgery. This technique of draping is highly economical and well suited for the

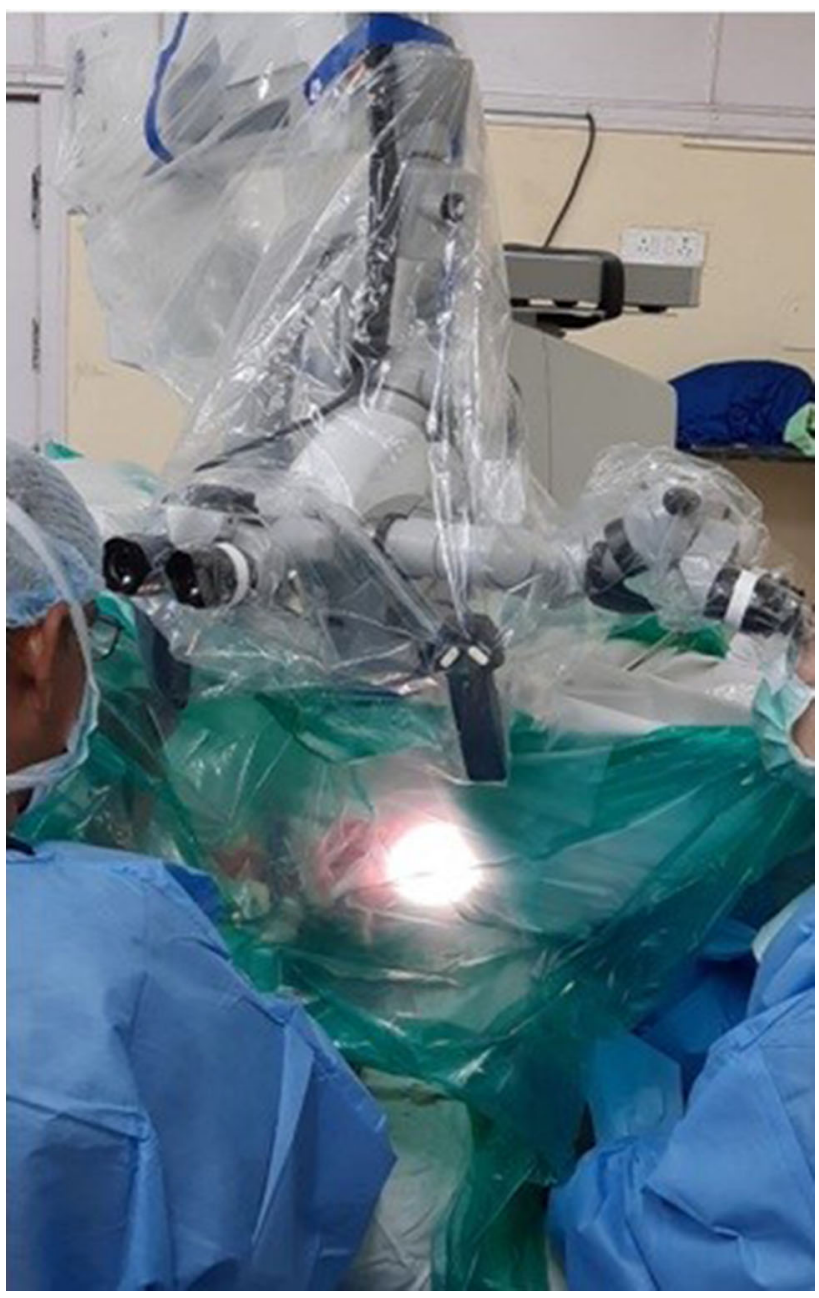

Fig. 2 Showing the Surgeon operating by insinuating his hands below the surgical drape

Indian scenario even at the most peripheral health care centres, as standard products used in otological surgeries were utilised to create the assembly. British society of otology has recently devised a technique using two microscope covers to minimise aerosol generation in mastoidectomy [1]. The technique, also simple to execute shows promise. However, in their technique, the second microscope cover used to drape the patient had an excess of length which appeared to gather at the area where the surgeon insinuated his hands under the drapes. We presume that, this may hamper the surgeon's manoeuvrability. The technique carried out by Carron et al. [2] on a cadaveric temporal bone excellently demonstrates containment of droplet soiling with the help of methylene blue dye. The technique however, comes across as slightly technically demanding and it's feasibility on live surgeries has yet not been commented upon.

The Covid-19 pandemic has changed the surgical protocols in its entirety. With newer protocols generated every 
day for combating the dissemination of viral particles, it is our commitment as otolaryngologists to contribute in devising an innovative draping system to perform aerosol generating surgeries to protect not only the surgeons but the nursing staff members, anaesthetists and the technicians present in the operation theatre. It goes without saying that, the concerned health personnel should also be equipped with appropriate PPEs and N-95 masks based on the risks involved in case to case basis.

We concur that this draping technique is effective in mitigating aerosols and recommend it for all otologic aerosol generating procedures.

Funding None.

\section{Compliance with Ethical Standards}

Conflict of interest Authors declare that they have no conflicts of interest.This is an innovative technique for draping to avoid aerosols during covid pandemic and describes the technique in detail.
Informed consent An informed consent including a covid consent was taken from the patient in whom this innovative technique was employed.

\section{References}

1. ENTUK 2020, Mastoidectomy in the COVID Era- The 2 Microscope Drape Method to Reduce Aerosolization, British Society of Otology, accessed 12 June 2020, https://www.entuk.org/sites/ default/files/Mastoidectomy\%2520in\%2520the\%2520COVID\% 2520Era\%2520\%E2\%80\%93\%2520The\%25202.pdf

2. Carron JD, Buck LS, Harbarger CF, Eby TL (2020) A simple technique for droplet control during mastoid surgery. JAMA Otolaryngol Head Neck Surg. https://doi.org/ 10.1001/jamaoto.2020.1064

Publisher's Note Springer Nature remains neutral with regard to jurisdictional claims in published maps and institutional affiliations. 